Pacific Journal of Mathematics

A NONLINEAR ELLIPTIC BOUNDARY VALUE PROBLEM 


\section{A NONLINEAR ELLIPTIC BOUNDARY VALUE PROBLEM}

\section{S. A. Williams}

This paper proves that there is a (weak) solution $u$ (not necessarily unique) to the generalized Dirichlet problem (with null boundary data) for the equation $A u+p u=h$. Here $A$ is a strongly and uniformly elliptic operator of order $2 m$ on a bounded open set $\Omega \subseteq R^{n}$. Also $A$ is "normal": roughly, $A A^{*}=A^{*} A$. The functions $p$ and $h$ are bounded and continuous, but are allowed to depend on $x(x \in \Omega), u$, and the generalized derivatives of $u$ up to order $m$. The values of $p$ are restricted to lie in a closed disk of the complex plane which contains the negative of no weak eigenvalue of $A$.

In [4], E. Landesman and A. Lazer proved that the boundary value problem

$$
\begin{aligned}
L u+p\left(x, u, \frac{\partial u}{\partial x_{1}}, \cdots, \frac{\partial u}{\partial x_{n}}\right) u & =h\left(x, u, \frac{\partial u}{\partial x_{1}}, \cdots, \frac{\partial u}{\partial x_{n}}\right) \text { on } D \\
u & =0 \text { on } \partial D
\end{aligned}
$$

has a (not necessarily unique) weak solution $u$. Here $D$ is any bounded open subset of $\boldsymbol{R}^{n}$ with boundary $\partial D$. Here $L$ is any linear, uniformly and strongly elliptic, self-adjoint, second order partial differential operator with only. second order terms and with real-valued, bounded measurable coefficients for its corresponding Dirichlet bilinear form. Here $p$ and $h$ are any real-valued, bounded, continuous functions. It is assumed that there exist constants $\gamma_{N}$ and $\gamma_{N+1}$ such that $\alpha_{N}<$ $\gamma_{N} \leqq p(z) \leqq \gamma_{N+1}<\alpha_{N+1}$ for every $z$ in $D \times \boldsymbol{R}^{n+1}$ (here $\alpha_{N}$ and $\alpha_{N+1}$ are the negatives of successive weak eigenvalues of $L$ ).

The present paper may perhaps best be viewed as a generalization of [4]. Although other generalizations are made, the main result is that the assumption that $L$ is self-adjoint can be replaced by the assumption that $L$ is "normal": roughly, $L L^{*}=L^{*} L$. Two examples at the end of the present paper show in what sense the result is best-possible and show that uniqueness can not be expected.

As in [4], the final existence result is proved using Schauder's theorem. In the solving of a preliminary linear problem, a contraction mapping and the fact that the spectral radius of a normal operator is equal to its norm replace the argument in [4] based on the maximun characterization of the eigenvalues and a comparison result for selfadjoint operators.

2. Notation. Let $Q$ be a bounded open subset of $\boldsymbol{R}^{n}$. Let 
$C_{0}^{\infty}(\Omega)$ denote the set of all infinitely differentiable complex-valued functions with compact support in $\Omega$. Let $L_{2}(\Omega)$ denote the Hilbert space of all complex-valued square-integrable functions on $\Omega$, with inner product (, ) and norm \|\| . Let $H^{(m)}(\Omega)$ denote the Hilbert space of all complex-valued functions on $\Omega$ whose distribution derivatives (using $C_{0}^{\infty}(\Omega)$ test functions) of order 0 through $m$ are in $L_{2}(\Omega)$. The inner product and norm of this space will be denoted by $(,)_{m}$ and \|\|$_{m}$ respectively. A multi-index is an $n$-tuple of nonnegative integers. If $\alpha=\left(\alpha_{1}, \alpha_{2}, \cdots, \alpha_{n}\right)$ is a multi-index, define

$$
|\alpha|=\alpha_{1}+\alpha_{2}+\cdots+\alpha_{n}
$$

and

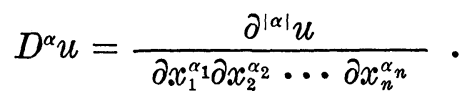

Here the indicated derivative is a distribution derivative. It will be used only when $u$ is in $H^{(|\alpha|)}(\Omega)$. Let $H_{0}^{(m)}(\Omega)$ denote the Hilbert subspace of $H^{(m)}(\Omega)$ obtained by taking the closure of the set $C_{0}^{\infty}(\Omega)$ in $H^{(m)}(\Omega)$.

Let $A$ be the formal differential operator given by

$$
A u=\sum_{\substack{|\alpha| \leq m \\|\beta| \leq m}}(-1)^{|\alpha|} D^{\alpha}\left(a_{\alpha \beta} D^{\beta} u\right),
$$

where the complex-valued functions $a_{\alpha \beta}$ are uniformly continuous in $\Omega$ for $|\alpha|=|\beta|=m$ and bounded and measurable otherwise. We assume that $A$ is uniformly strongly elliptic and normalized, i.e., that there exists a constant $E_{0}>0$ such that for all vectors $\xi=\left(\xi_{1}, \cdots, \xi_{n}\right)$ with real entries, and for all $x$ in $\Omega$,

$$
\operatorname{Re}\left\{\sum_{\substack{|>|=m \\|\beta|=m}} a_{\alpha \beta}(x) \xi_{1}^{\alpha_{1}+\beta_{1}} \xi_{2}^{\alpha_{2}+\beta_{2}} \cdots \xi_{n}^{\alpha_{n}+\beta_{n}}\right\} \geqq E_{0}|\xi|^{2 m}
$$

where Re takes the real part of any complex number and where $|\xi|$ denotes the length of $\xi$ in $\boldsymbol{R}^{n}$.

For any $\varphi$ and $\psi$ in $H_{0}^{(m)}(\Omega)$, define

$$
B[\varphi, \psi]=\sum_{\substack{|\alpha| \leq m \\|\beta| \leq m}}\left(D^{\alpha} \varphi, a_{\alpha \beta} D^{\beta} \psi\right)
$$

We say that $u$ is a solution of the generalized Dirichlet problem for $A u=f$ if and only if $f$ is in $L_{2}(\Omega), u$ is in $H_{0}^{(m)}(\Omega)$, and

$$
B[\varphi, u]=(\varphi, f) \text { for every } \varphi \text { in } H_{0}^{(m)}(\Omega) \text {. }
$$

We say that $\lambda$ is a weak eigenvalue for $A$ corresponding to weak eigenfunction $u$ if $u \neq 0$ is a solution of the generalized Dirichlet problem for $A u=\lambda u$. 
With the assumptions on $A$ made above, Garding's inequality holds (see S. Agmon [1], p. 102):

$$
\operatorname{Re} B[\phi, \phi]+\lambda_{0}(\phi, \phi) \geqq c_{0}\|\phi\|_{m}^{2} \text {. }
$$

Here $\lambda_{0}$ and $c_{0}$ are real constants with $c_{0}>0$. The inequality holds for each $\phi$ in $C_{0}^{\infty}(\Omega)$ and hence (taking limits in $H^{(m)}(\Omega)$ ) for each $\phi$ in $H_{0}^{(m)}(\Omega)$. For each $u$ in $H_{0}^{(m)}(\Omega)$, define

$$
\|u\|_{B}=\left[\operatorname{Re} B(u, u)+\lambda_{0}(u, u)\right]^{1 / 2} .
$$

An easy calculation shows that \|\|$_{B}$ is bounded above by a multiple of the \|\|$_{m}$ norm. Since Garding's inequality shows that it is also bounded below, these two norms on $H_{0}^{(m)}(\Omega)$ are equivalent.

We are assured by $[1 ; \mathrm{p} .102]$ that the generalized Dirichlet problem for $A u=f-\lambda_{0} u$ has for each $f$ in $L_{2}(\Omega)$ a unique solution $T_{0} f$ in $H_{0}^{(m)}(\Omega)$. The mapping $T_{0}: L_{2}(\Omega) \rightarrow H_{0}^{(m)}(\Omega)$ is linear and continuous.

Let $\mathscr{I}: H_{0}^{(m)}(\Omega) \rightarrow L_{2}(\Omega)$ denote the inclusion map and let $I: L_{2}(\Omega) \rightarrow$ $L_{2}(\Omega)$ denote the identity map.

3. Preliminary lemmas. Lemma 1, of interest in itself, greatly simplifies the proof of Theorem 2. Lemma 2 gives an elementary proof of the fact that the operator norm of a normal operator is equal to its spectral radius. Lemma 3 gives conditions under which a differential operator is "normal" in the sense required by this paper. Lemma 4 introduces an operator $T$ and Lemma 5 finds an upper bound for $\|\mathscr{I} T\|$. These last two lemmas will be used immediately in Theorem 1.

LemMa 1. $T_{0}$ is compact as a map from $L_{2}(\Omega)$ to $H_{0}^{(m)}(\Omega)$.

Proof. Let $\left\{f_{k}\right\}$ be a sequence in $L_{2}(\Omega)$ with $\left\|f_{k}\right\| \leqq r$. Since $\Omega$ is bounded, N. Dunford and J. Schwartz [3; p. 1693] assure us that $\mathscr{J}$ is compact. There is therefore a subsequence $\left\{g_{l}\right\}$ of $\left\{f_{k}\right\}$ such that $\left\{\mathscr{J} T_{0} g_{l}\right\}$ converges in $L_{2}(\Omega)$. Use $f=g_{l}-g_{k}$ and $\phi=T_{0} g_{l}-T_{0} g_{k}$ and the definition of $T_{0}$ to obtain

$$
\begin{aligned}
\left\|T_{0} g_{l}-T_{0} g_{k}\right\|_{B}^{2} & =\operatorname{Re} B\left[\phi, T_{0} f\right]+\lambda_{0}(\phi, \phi) \\
& \leqq\left|B\left[\phi, T_{0} f\right]+\lambda_{0}(\phi, \phi)\right| \\
& =\left|(\phi, f)-\lambda_{0}\left(\phi, T_{0} f\right)+\lambda_{0}(\phi, \phi)\right| \\
& =|(\phi, f)| \leqq\|f\|\|\phi\| \\
& \leqq 2 r\left\|T_{0} g_{l}-T_{0} g_{k}\right\| .
\end{aligned}
$$

Since $\left\{T_{0} g_{l}\right\}$ is a Cauchy sequence in $L_{2}(\Omega),\left\{T_{0} g_{l}\right\}$ is a Cauchy sequency 
in $H_{0}^{(m)}(\Omega)$ with the \|\|$_{B}$ norm. Therefore it is Cauchy under the \|\|$_{m}$ norm. ${ }^{1}$

LEMma 2. If $N$ is a normal operator in a Hilbert space with inner product (, ) and norm \|\| , then $\|N\|$, the operator norm of $N$, is equal to its spectral radius.

Proof. For any $x$ in the Hilbert space, $\left(N^{2} x, N^{2} x\right)=\left(N^{*} N x, N^{*} N x\right)$ and thus $\left\|N^{2}\right\|=\left\|N^{*} N\right\|$. But for any operator in a Hilbert space, $\left\|N^{*} N\right\|=\|N\|^{2}$ (see [3], p. 874). Thus $\left\|N^{2}\right\|=\|N\|^{2}$. By induction $\left\|N^{p}\right\|=\|N\|^{p}$ whenever $p$ is a power of 2 . The spectral radius of $N$ is given by the expression

$$
\lim _{p \rightarrow \infty}\left\|N^{p}\right\|^{1 / p} \quad \text { (see [3], p. 864) . }
$$

Considering the subsequence involving only those $p$ which are powers of 2 , the result follows. ${ }^{2}$

LEMMA 3. Let $A$ be a differential operator with coefficients having enough continuous derivatives so that $A^{*}, A A^{*}$, and $A^{*} A$ make sense classically on $C_{0}^{\infty}(\Omega)$. Suppose that $A A^{*}=A^{*} A$. Then $\mathscr{I} T_{0}$ is a normal operator.

Proof. The discussion in [1; pp. 97-103] shows that the generalized Dirichlet problem for $A^{*} u=f-\lambda_{0} u$ has for every $f$ in $L_{2}(\Omega)$ a unique solution $T_{0}^{*} f$ in $H_{0}^{(m)}(\Omega)$, where $\lambda_{0}$ is the same constant as was used to define $T_{0}$. For $\varphi$ and $\psi$ in $C_{0}^{\infty}(\Omega)$ the Dirichlet form for $A$ is given by $B[\varphi, \psi]=B_{A}[\varphi, \psi]=(\varphi, A \psi)$. Similarly $B_{A^{*}}[\varphi, \psi]=\left(\varphi, A^{*} \psi\right)$. It follows easily that $\mathscr{J} T_{0}^{*}$ is the adjoint of $\mathscr{J} T_{0}$.

The Dirichlet form for $\left(A+\lambda_{0}\right)^{*}\left(A+\lambda_{0}\right)$ is given by

$$
B_{\left(A+\lambda_{0}\right) *\left(A+\lambda_{0}\right)}[\varphi, \psi]=\left(\varphi,\left(A+\lambda_{0}\right) *\left(A+\lambda_{0}\right) \psi\right)=\left(\left[A+\lambda_{0}\right] \varphi,\left[A+\lambda_{0}\right] \psi\right) .
$$

An easy calculation shows that the Dirichlet form for $\left(A+\lambda_{0}\right)\left(A+\lambda_{0}\right)$ * is the same since $A A^{*}=A^{*} A$. If $u$ is a solution of the generalized. Dirichlet problem for $\left(\mathrm{A}+\lambda_{0}\right)^{*}\left(A+\lambda_{0}\right) u=0$, then

$$
\left(\left[A+\lambda_{0}\right] u,\left[A+\lambda_{0}\right] u\right)=0,
$$

so $\left(A+\lambda_{0}\right) u=0$ and hence finally $u=0$. By the Fredholm alternative the generalized Dirichlet problem for $\left(A+\lambda_{0}\right) *\left(A+\lambda_{0}\right) u=f$ has a unique solution $u$ in $H_{0}^{(2 m)}(\Omega)$. It is easy to see that $\mathscr{I} T_{0}^{*} \mathscr{J} T_{0} f=$ $u=\mathscr{J} T_{0} \mathscr{F} T_{0}^{*} f$. Thus $\mathscr{F} T_{0}^{*} \mathscr{F} T_{0}=\mathscr{J} T_{0} \mathscr{F} T_{0}^{*}$.

1 The proof of this lemma is motivated by a similar calculation in [4; pp. 321, 322].

2. The author wishes to thank Dr. S. Ebenstein for his elementary proof of Lemma 2. 
LEMMA 4. If $\gamma_{0}$ is a complex number such that $-\gamma_{0}$ is not a weak eigenvalue of $A$, then we may set $T=T_{0}\left[\left(\gamma_{\theta}-\lambda_{0}\right) \mathscr{F} T_{0}+I\right]^{-1}$ and have for every $f$ in $L_{2}(\Omega)$ and every $\varphi$ in $H_{0}^{(m)}(\Omega)$ that

$$
B[\varphi, T f]+\overline{\gamma_{0}}(\varphi, T f)=(\varphi, f) .
$$

(Thus Tf is the unique weak solution of $A u+\gamma_{0} u=f$. )

Proof. Since $-\gamma_{0}$ is not a weak eigenvalue of $A,\left(\lambda_{0}-\gamma_{0}\right)^{-1}$ is not an eigenvalue of $\mathscr{J} T_{0}$. Since $\mathscr{J} T_{0}$ is compact, every nonzero complex number in its spectrum must be an eigenvalue. Therefore $\left(\lambda_{0}-\gamma_{0}\right)^{-1}$ is not in the spectrum of $\mathscr{J} T_{0}$, so $\left[\mathscr{J} T_{0}-\left(\lambda_{0}-\gamma_{0}\right)^{-1} I\right]^{-1}$ (and hence $\left.\left[\left(\gamma_{0}-\lambda_{0}\right) \mathscr{J} T_{0}+I\right]^{-1}\right)$ exists and is "continuous.

$$
\begin{aligned}
B[\varphi, T f]+ & \bar{\gamma}_{0}(\varphi, T f) \\
= & -\lambda_{0}\left(\varphi, T_{0}\left[\left(\gamma_{0}-\lambda_{0}\right) \mathscr{F} T_{0}+I\right]^{-1} f\right)+\left(\varphi,\left[\left(\gamma_{0}-\lambda_{0}\right) \mathscr{F} T_{0}+I\right]^{-1} f\right) \\
& \quad+\bar{\gamma}_{0}\left(\varphi, T_{0}\left[\left(\gamma_{0}-\lambda_{0}\right) \mathscr{I} T_{0}+I\right]^{-1} f\right) \\
= & \left(\varphi,\left[\left(\gamma_{0}-\lambda_{0}\right) \mathscr{F} T_{0}+I\right]\left[\left(\gamma_{0}-\lambda_{0}\right) \mathscr{I} T_{0}+I\right]^{-1} f\right)=(\varphi, f) .
\end{aligned}
$$

LEMMA 5. Assume that $\mathscr{J} T_{0}$ is a normal operator and that $\left|z-\gamma_{0}\right| \leqq c$ is a disk in the complex plane which contains the negative of no weak eigenvalue of $A$. Then $\|\mathscr{I} T\| c<1$, where $T$ is the map of the above lemma.

Proof. Since $\mathscr{J} T_{0}$ is a normal operator, so is $\left[\left(\gamma_{0}-\lambda_{0}\right) \mathscr{I} T_{0}+I\right]^{-1}$. Since $\mathscr{J} T_{0}$ and this operator commute,

$$
\mathscr{J} T=\mathscr{J} T_{0}\left[\left(\gamma_{0}-\lambda_{0}\right) \mathscr{J} T_{0}+I\right]^{-1}
$$

is normal. Therefore $\|\mathscr{J} T\|$ is the same as the spectral radius of $\mathscr{J} T$. Since $\mathscr{J} T$ is compact, the spectral radius is the supremum of the norms of the eigenvalues of $\mathscr{J} T$. But $\lambda$ is a weak eigenvalue of $A$ if and only if $\left(\lambda+\gamma_{0}\right)^{-1}$ is an eigenvalue of $\mathscr{T} T$. Thus the weak eigenvalues of $A$ have no accumulation point in the (finite) complex plane. Since $\left|-\lambda-\gamma_{0}\right| \geqq c+\varepsilon$ for some $\varepsilon>0$ and every weak eigenvalue $\lambda$ of $A,\left|\left(\lambda+\gamma_{0}\right)^{-1}\right| \leqq(c+\varepsilon)^{-1}$ so that every eigenvalue of $\mathscr{J} T$ has norm $\leqq(c+\varepsilon)^{-1}$. Thus $\|\mathscr{J} T\| c<1$ as claimed.

\section{The preliminary linear problem.}

THEOREM 1. Let $D$ be a closed disk $\left\{z \in C ;\left|z-\gamma_{0}\right| \leqq c\right\}$ in the complex plane which contains the negative of no weak eigenvalue of $A$. Let $h$ be in $L_{2}(\Omega)$ and let $p$ be a measurable function on $\Omega$ whose values lie in the disk $D$. Suppose that the operator $\mathscr{I} T_{0}$ associated with $A$ is "normal. Then the generalized Diriahlet problem 
for $A u+p u=h$ has a unique solution $u$ in $H_{0}^{(m)}(\Omega)$. Moreover, there exists a constant $M$ independent of $p$ such that

$$
\operatorname{Re} B[u, u]+\lambda_{0}(u, u) \leqq M(h, h) \cdot
$$

Proof. We want $A u+p u=h$, or equivalently $A u+\gamma_{0} u=h-$ $\left(p-\gamma_{0}\right) u$. Thus we want $u=T\left(h-\left(p-\gamma_{0}\right) u\right)$, where $T$ is the map of Lemmas 4 and 5 . We prove that the map from $L_{2}(\Omega)$ into itself given by $u \rightarrow \mathscr{J} T\left[h-\left(p-\gamma_{0}\right) u\right]$ is a contraction map.

For any $u_{1}$ and $u_{2}$ in $L_{2}(\Omega)$,

$$
\begin{aligned}
& \mathscr{J} T\left[h-\left(p-\gamma_{0}\right) u_{1}\right]-\mathscr{F} T\left[h-\left(p-\gamma_{0}\right) u_{2}\right] \| \\
& =\left\|\mathscr{F} T\left(p-\gamma_{0}\right)\left(u_{1}-u_{2}\right)\right\| \leqq\|\mathscr{F} T\| c\left\|u_{1}-u_{2}\right\| \cdot
\end{aligned}
$$

Since $\|\mathscr{I} T\| c<1$ by Lemma 5 , the map is a contraction as claimed. Thus there exists a unique $v$ in $L_{2}(\Omega)$ such that $v=\mathscr{J} T\left[h-\left(p-\gamma_{0}\right) v\right]$.

Let $Q=\|\mathscr{J} T\|(1-\|\mathscr{J} T\| c)^{-1}$. Then $Q=\|\mathscr{J} T\|+\|\mathscr{J} T\| c Q$. Since $\|u\| \leqq Q\|h\|$ implies that

$$
\begin{aligned}
\left\|\mathscr{F} T\left[h-\left(p-\gamma_{0}\right) u\right]\right\| & \leqq\|\mathscr{J} T\|\|h\|+c\|\mathscr{F} T\|\|u\| \\
& \leqq\|\mathscr{F} T\|\|h\|+c\|\mathscr{F} T\| Q\|h\| \\
& =Q\|h\|,
\end{aligned}
$$

it follows that for fixed $h$ the ball $\left\{u \in L_{2}(\Omega)\right.$; $\left.\|u\| \leqq Q\|h\|\right\}$ is mapped into itself by our contraction map. Therefore the fixed point $v$ satisfies $\|v\| \leqq Q\|h\|$. Since the \|\|$_{m}$ norm and the \|\|$_{B}$ norm are equivalent, and since

$$
\|v\|_{m}=\left\|T\left[h-\left(p-\gamma_{0}\right) v\right]\right\|_{m} \leqq\|T H\| h-\left(p-\gamma_{0}\right) v \|,
$$

(here $\|T\|$ is the operator norm of $T: L_{2}(\Omega) \rightarrow H_{0}^{(m)}(\Omega)$ ) it follows easily that there exists an $M$ such that $\|v\|_{B}^{2} \leqq M\|h\|^{2}$.

\section{The nonlinear problem.}

THeOREM 2. Let $D$ be a closed disk in the complex plane which contains the negative of no weak eigenvalue of $A$. Let $h\left(x, u, \partial u / \partial x_{1}, \cdots\right)$ and $p\left(x, u, \partial u / \partial x_{1}, \cdots\right)$ be continuous functions of their arguments, allowed to involve derivatives of $u$ up to order $m$. Let $|h(x, u, \cdots)| \leqq r$ and assume that the values of $p$ are always in the disk D. Assume that the operator $\mathscr{J} T_{0}$ associated with $A$ is normal. Then the generalized Dirichlet problem for

$$
A u+p\left(x, u, \frac{\partial u}{\partial x_{1}}, \cdots\right) u=h\left(x, u, \frac{\partial u}{\partial x_{1}}, \cdots\right)
$$

has a (not necessarily unique) solution $u$ in $H_{0}^{(m)}(\Omega)$. 
Proof. Define a map $G: H_{0}^{(m)}(\Omega) \rightarrow H_{0}^{(m)}(\Omega)$ as follows: for every $u$ in $H_{0}^{(m)}(\Omega)$, let $G(u)$ be the unique solution $v$ in $H_{0}^{(m)}(\Omega)$ of

$$
v=\mathscr{J} T\left[h\left(x, u, \frac{\partial u}{\partial x_{1}}, \cdots\right)-\left(p\left(x, u, \frac{\partial u}{\partial x_{1}}, \cdots\right)-\gamma_{0}\right) v\right],
$$

where $\gamma_{0}$ is the center of the disk $D$ and $T$ is the operator of Lemmas 4 and 5. It is clear that a fixed point of $G$ would furnish a solution for the generalized Dirichlet problem for (3). We will show that $G$ is continuous and compact from a bounded, closed, convex subset $S$ of $H_{0}^{(m)}(\Omega)$ into itself. Schauder's theorem (see, for example, J. Cronin [2], p. 131) then assures us a fixed point.

Since $|h(x, u, \cdots)| \leqq r,(h, h) \leqq R=r^{2}$ meas $(\Omega)<\infty$. Using the constant $M$ of Theorem 1, $\|G(u)\|_{B}^{2} \leqq M R$ for all $u$ in $H_{0}^{(m)}(\Omega)$. Thus if we take $S=\left\{u \in H_{0}^{(m)}(\Omega)\right.$; $\left.\|u\|_{B}^{2} \leqq M R\right\}, S$ is a bounded, closed, convex set of $H_{0}^{(m)}(\Omega)$ and $G(S) \subseteq S$.

Now we show that $G$ is continuous. Let $\left\{u_{k}\right\}$ be a sequence in $H_{0}^{(m)}(\Omega)$ converging to $u$. The sequence $\left\{h\left(x, u_{k}, \cdots\right)-\left(p\left(x, u_{k}, \cdots\right)-\right.\right.$ $\left.\left.\gamma_{0}\right) G\left(u_{k}\right)\right\}$ is clearly bounded in $L_{2}(\Omega)$, so since $T$ is compact (Lemma 1 shows that $T_{0}$ is compact, and $T$ is $T_{0}$ composed with a continuous map) there is a subsequence of $\left\{G\left(u_{k}\right)\right\}$ which converges in $H_{0}^{(m)}(\Omega)$ to a limit $v$. Then taking limits with the corresponding subsequence of $\left\{u_{k}\right\}$,

$$
v=\mathscr{J} T\left[h(x, u, \cdots)-\left(p(x, u, \cdots)-\gamma_{0}\right) v\right],
$$

so that $v=G(u)$. Since any subsequence of $\left\{G\left(u_{k}\right)\right\}$ has a subsequence converging in $H_{0}^{(m)}(\Omega)$ to $G(u),\left\{G\left(u_{k}\right)\right\}$ itself converges in $H_{0}^{(m)}(\Omega)$ to $G(u)$, proving continuity.

Now we show that $G$ is compact. Let $\left\{u_{k}\right\}$ be a bounded sequence in $H_{0}^{(m)}(\Omega)$. Then the sequence $\left\{h\left(x, u_{k}, \cdots\right)-\left(p\left(x, u_{k}, \cdots\right)-\gamma_{0}\right) G\left(u_{k}\right)\right\}$ is bounded in $L_{2}(\Omega)$, so the fact that $T$ is compact assures us a subsequence of $\left\{G\left(u_{k}\right)\right\}$ which converges in $H_{0}^{(m)}(\Omega)$.

\section{Examples and a remark.}

EXAMPLE 1. If the disk $D$ includes the negative of a weak eigenvalue $\lambda$ of $A$, let $v$ be a weak eigenfunction of $A^{*}$ corresponding to the weak eigenvalue $\bar{\lambda}$. If $h(x)$ is any bounded continuous function on $\Omega$ such that $(h, v) \neq 0$, then the generalized Dirichlet problem for $A u+\lambda u=h$ has no solution, since the Fredholm alternative applies [1, p. 102]. It is in this sense that Theorem 2 is best possible.

ExAmple 2. Suppose that there is a weak eigenvalue $\lambda$ of $A$ which corresponds to a continuous weak eigenfunction $v$ with $|v(x)| \leqq 1$ for every $x$ in $\Omega$. Let $\gamma_{0}$ be the center of the disk $D$ and let $p=\gamma_{0}$ 
identically. Let $h=h(u)$ be a bounded $C^{\infty}$ function of $u$ with $h(u)=$ $\gamma_{0} u+\lambda u$ for $|u| \leqq 1$. Then $v$ and $v / 2$ are two distinct solutions of the generalized Dirichlet problem for $A u+p u=h$. This shows that we cannot expect a unique solution to problems of the type discussed in this paper.

REMARK. Consider the generalized Dirichlet problem for $A u=$ $f\left(x, u, \partial u / \partial x_{1}, \cdots\right)$, where $f$ is a continuous function of its arguments, involving derivatives of $u$ up to order $m$. Under what circumstances can we write $f=-p u+h$, where $|h| \leqq r$ and the values of $p$ lie in a closed disk $D$ with center $\gamma_{0}$ and radius $c$ ? Clearly $\left|f+\gamma_{0} u\right| \leqq$ $c|u|+r$ is a necessary condition. It is interesting to note that this condition is also sufficient. To see this, given an $f$ satisfying this growth condition, define $p$ to be the closest point in $D$ to $-f / u$ for any values of the arguments with $|u| \geqq 1$. Then extend $p$ so as to be defined also for $|u|<1$, so as to be continuous overall, and so as to have each of its values in $D$. Then set $h=f+p u$. (For $|u| \geqq 1$ we have $|h| \leqq r$, but for $|u|<1$, although $h$ as given in the above construction is bounded, we are not assured that $|h| \leqq r$.)

\section{REFERENCES}

1. S. Agmon, Lectures on Elliptic Boundary Value Problems, Van Nostrand, New York, 1965.

2. J. Cronin, Fixed points and topological degree in nonlinear analysis, Math. Surveys, No. 11, A.M.S., Providence, 1964.

3. N. Dunford and J. Schwartz, Linear Operators, Part 2, John Wiley, New York, 1963.

4. E. Landesman and A. Lazer, Linear eigenvalues and a nonlinear boundary value problem, Pacific J. of Math., 33 (1970), 311-328.

Received September 13, 1971.

WAYNe State UNIVERSITY 


\section{PACIFIC JOURNAL OF MATHEMATICS}

\section{EDITORS}

\section{H. SAMELSON}

Stanford University

Stanford, California 94305

C. R. Новву

University of Washington Seattle, Washington 98105

\section{J. DuGundJI}

Department of Mathematics University of Southern California Los Angeles, California 90007

RICHARD ARENS

University of California Los Angeles, California 90024

\section{ASSOCIATE EDITORS}
E. F. BECKENBACH
B. H. NeumanN
F. WOLF
K. YoSHIDA

\section{SUPPORTING INSTITUTIONS}

\author{
UNIVERSITY OF BRITISH COLUMBIA \\ CALIFORNIA INSTITUTE OF TECHNOLOGY \\ UNIVERSITY OF CALIFORNIA \\ MONTANA STATE UNIVERSITY \\ UNIVERSITY OF NEVADA \\ NEW MEXICO STATE UNIVERSITY \\ OREGON STATE UNIVERSITY \\ UNIVERSITY OF OREGON \\ OSAKA UNIVERSITY
}

\author{
UNIVERSITY OF SOUTHERN CALIFORNIA \\ STANFORD UNIVERSITY \\ UNIVERSITY OF TOKYO \\ UNIVERSITY OF UTAH \\ WASHINGTON STATE UNIVERSITY \\ UNIVERSITY OF WASHINGTON \\ $*{ }^{*}$
AMERICAN MATHEMATICAL SOCIETY
NAVAL WEAPONS CENTER
}

The Supporting Institutions listed above contribute to the cost of publication of this Journal, but they are not owners or publishers and have no responsibility for its content or policies.

Mathematical papers intended for publication in the Pacific Journal of Mathematics should be in typed form or offset-reproduced, (not dittoed), double spaced with large margins. Underline Greek letters in red, German in green, and script in blue. The first paragraph or two must be capable of being used separately as a synopsis of the entire paper. The editorial "we" must not be used in the synopsis, and items of the bibliography should not be cited there unless absolutely necessary, in which case they must be identified by author and Journal, rather than by item number. Manuscripts, in duplicate if possible, may be sent to any one of the four editors. Please classify according to the scheme of Math. Rev. Index to Vol, 39. All other communications to the editors should be addressed to the managing editor, Richard Arens, University of California, Los Angeles, California, 90024.

50 reprints are provided free for each article; additional copies may be obtained at cost in multiples of 50 .

The Pacific Journal of Mathematics is issued monthly as of January 1966. Regular subscription rate: $\$ 48.00$ a year (6 Vols., 12 issues). Special rate: $\$ 24.00$ a year to individual members of supporting institutions.

Subscriptions, orders for back numbers, and changes of address should be sent to Pacific Journal of Mathematics, 103 Highland Boulevard, Berkeley, California, 94708.

PUBLISHED BY PACIFIC JOURNAL OF MATHEMATICS, A NON-PROFIT CORPORATION

Printed at Kokusai Bunken Insatsusha (International Academic Printing Co., Ltd.), 270, 3-chome Totsuka-cho, Shinjuku-ku, Tokyo 160, Japan. 


\section{Pacific Journal of Mathematics}

\section{Vol. 44, No. $2 \quad$ June, 1973}

Tsuyoshi Andô, Closed range theorems for convex sets and linear liftings . . . . . . 393

Richard David Bourgin, Conically bounded sets in Banach spaces . . . . . . . . . 411

Robert Jay Buck, Hausdorff dimensions for compact sets in $R^{n} \ldots \ldots \ldots \ldots \ldots \ldots . \ldots 421$

Henry Cheng, A constructive Riemann mapping theorem ................ 435

David Fleming Dawson, Summability of subsequences and stretchings of

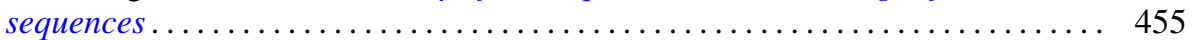

William Thomas Eaton, A two sided approximation theorem for 2-spheres ....... 461

Jay Paul Fillmore and John Herman Scheuneman, Fundamental groups of compact complete locally affine complex surfaces ....................... 487

Avner Friedman, Bounded entire solutions of elliptic equations . . . . . . . . . . . 497

Ronald Francis Gariepy, Multiplicity and the area of an $(n-1)$ continuous

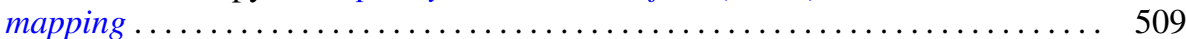

Andrew M. W. Glass, Archimedean extensions of directed interpolation groups . . . . 515

Morisuke Hasumi, Extreme points and unicity of extremum problems in $H^{1}$ on

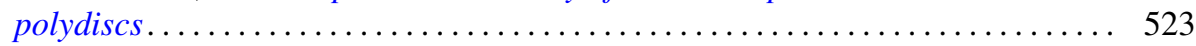

Trevor Ongley Hawkes, On the Fitting length of a soluble linear group . . . . . . 537

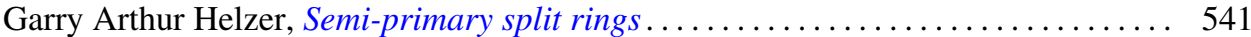

Melvin Hochster, Expanded radical ideals and semiregular ideals . . . . . . . . . 553

Keizō Kikuchi, Starlike and convex mappings in several complex variables . . . . . . 569

Charles Philip Lanski, On the relationship of a ring and the subring generated by its

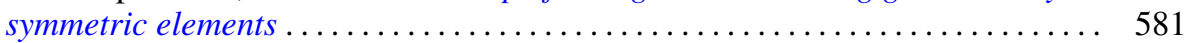

Jimmie Don Lawson, Intrinsic topologies in topological lattices and semilattices ........................................... 593

Roy Bruce Levow, Counterexamples to conjectures of Ryser and de Oliveira ...... 603

Arthur Larry Lieberman, Some representations of the automorphism group of an infinite continuous homogeneous measure algebra ..........

William George McArthur, $G_{\delta}$-diagonals and metrization theorems $\ldots .$.

James Murdoch McPherson, Wild arcs in three-space. II. An invariant of

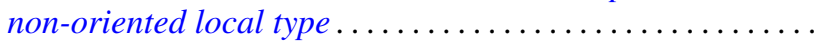

H. Millington and Maurice Sion, Inverse systems of group-valued measures ...

C. Edward Moore, Concrete semispaces and lexicographic separation of convex

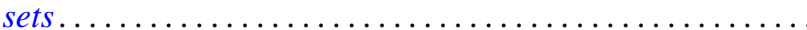

Jingyal Pak, Actions of torus $T^{n}$ on $(n+1)$-manifolds $M^{n+1}$.

Merrell Lee Patrick, Extensions of inequalities of the Laguerre and Turán type . . . . 675

Harold L. Peterson, Jr., Discontinuous characters and subgroups of finite index. . . . 683

S. P. Philipp, Abel summability of conjugate integrals . . . . . . . . . . . . . 693

R. B. Quintana and Charles R. B. Wright, On groups of exponent four satisfying an

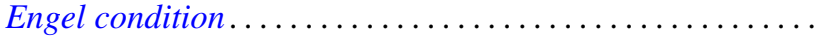

Marlon C. Rayburn, On Hausdorff compactifications. . . . . . . . . .

Martin G. Ribe, Necessary convexity conditions for the Hahn-Banach theorem in

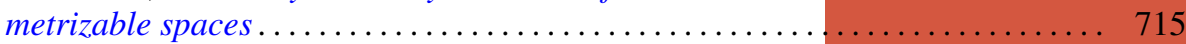

Ryōtarō Satō, On decomposition of transformations in infinite measure spaces .... 733

Peter Drummond Taylor, Subgradients of a convex function obtained from a

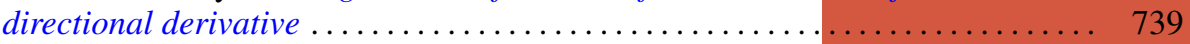

James William Thomas, A bifurcation theorem for $k$-set contractions . . . . . . . . 749 Clifford Edward Weil, A topological lemma and applications to real functions . . . . 757

Stephen Andrew Williams, A nonlinear elliptic boundary value problem . . ....... 767

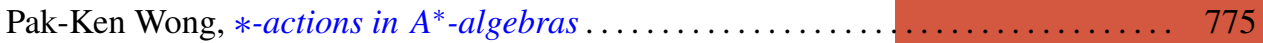

